\title{
A CONDITION FOR THE EXISTENCE OF A STRONGLY EMBEDDED SUBGROUP
}

\author{
MICHAEL ASCHBACHER ${ }^{1}$
}

\begin{abstract}
Let $D$ be a normal set of involutions in a finite group. Form the involutory graph with vertex set $D$ by joining distinct commuting elements of $D$. Assume the product of any two such elements is in $D$, and the graph is disconnected. Then the group generated by $D$ contains a strongly embedded subgroup. Two corollaries are proved.
\end{abstract}

Let $G$ be a finite group and $D$ a collection of involutions normalized by $G$. Form the graph $\mathscr{D}=\mathscr{D}(D)$ with vertex set $D$ and edges $(u, v)$ where $u v=v u \neq 1$.

Theorem 1. Assume $\mathscr{Z}$ is disconnected and for any edge $(u, v)$ in $\mathscr{D}$, $u v \in D$. Let $H$ be the normalizer in $G$ of a connected component of $\mathscr{D}$. Then $H \cap\langle D\rangle$ is strongly embedded in $\langle D\rangle$.

The following two theorems are corollaries to Theorem 1 and Bender's classification of groups with a strongly embedded subgroup.

THEOREM 2. Let $G$ be a transitive permutation group on a set $\Omega$, let $x \in \Omega$, and let $H=G_{\alpha}$. Assume $Q$ is a normal subgroup of even order in $H$ such that every involution $z$ in $Q$ fixes exactly one point of $\Omega$. Set $L=\left\langle z^{G}\right\rangle$. Then either

(1) $L \leqq Z^{*}(G)$ and $O(L)$ is transitive on $\Omega$, or

(2) $L^{S 2}$ is $L_{2}(q), S z(q)$, or $U_{3}(q), q>2$ even, in its natural doubly transitive representation.

THEOREM 3. Let $z^{(i}$ be a conjugacy class of involutions in the group $G$, set $W=\left\langle z^{G} \cap C(z)\right\rangle$ and $L=\left\langle z^{\left({ }^{*}\right.}\right\rangle$. Assume $W$ is abelian and $W^{\#}$ is fused in $G$ and $W \Varangle G$. Then either

(1) $L \leqq Z^{*}(G)$, or

(2) $L$ is isomorphic to $L_{2}(q), S z(q)$, or $U_{3}(q), q>2$ even, or the perfect central extension of $Z_{3}$ by $U_{3}(q), q \equiv-1 \bmod 3$ even.

Presented to the Society, January 26, 1973 under the title Generalized strongly embedded subgroups; received by the editors July 31, 1972.

AMS (MOS) subject classifications (1970). Primary 20D05.

${ }^{1}$ This research was supported in part by NSF GP 25664.

c) American Mathematical Society 1973 
Theorem 3 can be used to significantly shorten the proof of an important result of Shult [5], classifying finite groups in which the weak closure of a central involution in its centralizer is abelian. Theorem 2 contains theorems of Shult ([5], [6]) and Hering [4] on transitive permutation groups.

The proof of Theorem 1 depends on the following four results:

THEOREM 4 (BENDER [2]). Let $H$ be a strongly embedded subgroup of the finite group $G$. Then either $G$ has 2-rank one or $O^{2}(G) / O(G)$ is isomorphic to $L_{2}(q), S z(q)$, or $U_{3}(q), q>2$ even.

Theorem 5 (Steinberg, Alperin and Gorenstein, Griess [3]). Let $G \cong L_{2}(q), S z(q)$, or $U_{3}(q), q>2$ even, and let $G^{*}$ be the covering group of $G$. Then $G=G^{*}$ except in the following cases:

(1) $G \cong L_{2}(4)$ and $G^{*} \cong S L_{2}(5)$,

(2) $G \cong S z(8)$ and $Z\left(G^{*}\right) \cong Z_{2} \times Z_{2}$,

(3) $G \cong U_{3}(q), q \equiv-1 \bmod 3$, and $Z\left(G^{*}\right) \cong Z_{3}$.

THEOREM 6 [1]. Let $G$ be a finite group, $H$ a proper subgroup of $G$ and $z$ an involution in the center of a Sylow 2-subgroup of G. Assume

(1) $z \in H^{g}$ if and only if $g \in H$, and

(2) if $u$ is an involution with $z \in C(u) \lesseqgtr H$ then $H \cap L$ is strongly embedded in $L=\left\langle z^{G} \cap C(u)\right\rangle$.

Then $H \cap\left\langle z^{G}\right\rangle$ is strongly embedded in $\left\langle z^{G}\right\rangle$.

Lemma 7. Assume the hypothesis of Theorem 1. Let $\Delta$ be the connected component of $\mathscr{D}$ normalized by $H$, let $z \in \Delta$, and let $S$ be a Sylow 2-subgroup of $C(z)$. Then

(1) $G^{D}$ is transitive,

(2) $z \in H^{g}$ if and only if $g \in H$,

(3) $S$ is Sylow in $G$.

Proof. Let $u \in D$ and assume $u z$ has even order. Let $v$ be the involution in $\langle u z\rangle$. Thus $v z \in D$, so by hypothesis $v=(v z) z \in D$. So $u, v$, and $z$ are all in $\Delta$.

Let $w \in D-\Delta$ and $x \in \Delta$. Then by the last paragraph, $w x$ and $w z$ have odd order, so $w$ is conjugate to $x$ in $\langle w, x\rangle$ and to $z$ in $\langle w, z\rangle$. Therefore $G^{I)}$ is transitive.

Assume $S$ is not Sylow in $G$. Then $S$ is of index 2 in some 2-subgroup $T$ of $G$. Let $t \in T-S$. Then $z \neq z^{t} \in Z(S)$, so $u=z z^{t} \in D \cap Z(T)$, impossible as $G^{D}$ is transitive. Notice that $z^{S}=z \in \Delta$, so $S \leqq H$.

Finally let $u \in H \cap D$. As $S$ is Sylow in $H$ we may assume $u \in S$. Thus $[u, z]=1$, so $u \in \Delta$. Then $H \cap D=\Delta$, implying (2).

Now for the proof of Theorem 1. Assume the theorem to be false and let $G$ be a minimal counterexample. First assume $x$ is an involution in 
the center of $G$, and set $\bar{G}=G /\langle x\rangle$. Let $(\bar{u}, \bar{v})$ be an edge in $\mathscr{D}(\bar{D})$. Then either $(u, v)$ is an edge in $\mathscr{D}$ and thus $u v \in D$, or $(u v)^{2}=x$. In the latter case $u x \in D$, so $x \in D$, impossible as $G^{D}$ is transitive. So $u v \in D$ and thus $\bar{u} \bar{v} \in \bar{D}$. It follows that $\bar{G}$ satisfies the hypothesis of Theorem 1 , so by minimality of $G, \bar{H} \cap \bar{L}$ is strongly embedded in $\bar{L}=\langle\bar{D}\rangle$. By Theorem 4 , $\bar{L} / O(\bar{L}) \cong Z_{2}, L_{2}(q), S z(q)$, or $U_{3}(q), q>2$ even. It follows from Theorem 5 that either $L=\langle x\rangle \times K$ with $K \cong \bar{L}$, or $L / O(L) \cong S L_{2}(5)$ or $(S z)^{\wedge}(8)$, a perfect extension of $\langle x\rangle$ by $S z(8) . L / O(L) \cong S L_{2}(5)$ as $L$ contains an involution $z \neq x$. If $L / O(L) \cong(S z)^{\wedge}(8)$ then $x=z u$ for some $u \in D$, so $x \in D$, a contradiction. So $L=\langle x\rangle \times K$. Further if $u$ and $v$ are distinct commuting involutions in $L-(K \cup\{x\})$, then $u v \notin D$, so $K=\langle D\rangle$. But $H \cap K$ is strongly embedded in $K$, a contradiction.

It follows that $Z(G)$ has odd order. Next suppose $u$ is an involution centralizing $z \in H \cap D$ with $C(u) \npreceq H$. Then $u \notin Z(G)$ so $C(u) \neq G$. But $C(u)$ satisfies the hypothesis of Theorem 1 , so minimality of $G$ implies $H \cap L$ is strongly embedded in $L=\langle C(u) \cap D\rangle$. But now Theorem 6 contradicts the choice of $G$.

Finally the proofs of Theorems 2 and 3. In Theorem 2, $H$ is the stabilizer of a point of $\Omega$; in Theorem 3 set $H=N(W)$. In Theorem 2 let $z$ be an involution in $Q$. Set $X=O(G)$ and $L=\left\langle z^{G}\right\rangle$. By Theorem $1, H \cap L$ is strongly embedded in $L$. We may assume $z \notin Z^{*}(G)$. Thus there exists a 4-group $U$ containing $z$ with $U^{\#} \subseteq z^{G}$. Then $X=\prod_{U} C_{X}(u) \leqq H$. Therefore in Theorem 2, $X$ fixes $\Omega$ pointwise, so $X=1$. And in Theorem 3, $[X, W] \leqq X \cap W=1$, so $X \cap L \leqq Z(L)$. Thus Theorems 4 and 5 yield the desired conclusion.

\section{REFERENCES}

1. M. Aschbacher, Finite groups with a proper 2-generated core (to appear).

2. H. Bender, Transitive Gruppen gerader Ordung in deven jede Involution genau einere Punkt festalt, J. Algebra 17 (1971), 527-554.

3. W. Feit, The current situation in the theory of finite simple groups, Proc. Internat. Congress Math. (Nice, 1970), vol. 1, Gauthier-Villars, Paris, 1971, pp. 55-93.

4. C. Hering, On subgroups with trivial normalizer intersection, J. Algebra 20 (1972), 622-629.

5. E. Shult, On the fusion of an involution in its centralizer (to appear).

6. - On a class of doubly transitive groups, Illinois J. Math. 16 (1972), 434-445.

Department of Mathematics, California Institute of Technology, Pasadena, CALIFornia 91109 